\title{
1 Assessing the relationship between cognitive empathy, age, and face 2 perception using a sequential Bayesian analysis
}

4 J.D. Kist, R.A.I. Bethlehem, Barnaby Stonier, Olivier Sluijters, S. K. Crockford(1,2) , Elke de

5 Jonge, Jan Freyberg, Simon Baron-Cohen, O. E. Parsons

7 Autism Research Centre, Psychiatry Department, Cambridge University, 2, Faculty of Modern and

8 Medieval Languages and Linguistics, Cambridge University

\section{Abstract}

The ability to correctly identify emotions in social stimuli such as faces is proposed to affect one's level of cognitive empathy. The Fusiform Face Area shows a heightened neural response during the perception of faces relative to objects. We tested whether neural responses to social stimuli were associated with performance in a measure of cognitive empathy, the 'Reading the Mind in the Eyes' Task. To quantify face perception, participants were presented with images during a fast serial presentation task which elicited a steady state visual evoked potential, measured using electroencephalography. A Sequential Bayesian Analysis was used to assess if face specific neural responses were associated with either cognitive empathy or age. Data were collected from a participant group of both neurotypical individuals and individuals on the autistic spectrum. We found no significant relationship between the face-specific neural signature, cognitive empathy or age. This

23 study highlights the efficiency of the Sequential Bayesian Analysis as an effective method of participant recruitment. 


\section{Introduction}

27 Autism spectrum condition (ASC) is a highly varied neurodevelopmental condition 28 characterized by differences in sensory perception, difficulties with social interactions and 29 the display of repetitive behaviors and obsessions (American Psychiatric Association 2013).

30 The atypical patterns found in the sensory perception in ASC have been suggested to affect 31 social cognition and consequently behavior in a bottom-up manner (Robertson and Baron32 Cohen 2017; Dellapiazza 2018). The ability to engage in successful social interaction relies 33 not only on bottom-up processes such as sensory perception, but also on more internal, 34 higher level processes such as cognitive empathy. Cognitive empathy is defined as the ability to recognize and understand mental states in oneself or other people. Levels of cognitive empathy vary throughout the entire population, along a bell curve, however autistic individuals are consistently found to have lower levels of cognitive empathy than non-autistic individuals (Jones et al. 2010; Rueda, Fernández-Berrocal, and Baron-Cohen 2015; Greenberg, Warrier, and Allison 2018).

The perception of emotion in other people is at the core of cognitive empathy: whether we perceive an emotion correctly in someone else determines how we react to them (Thye et al. 2018; S. Baron-Cohen 2011). A large amount of information concerning an individual's emotion and identity is contained in the face (Williams and Cross 2018; Simon Baron-Cohen et al. 1997). Because of this, the ability to read facial information quickly is an important, evolutionarily beneficial trait and is vital to facilitating social interactions (Walter et al. 2005); (Kanwisher and Yovel 2006). It has been suggested that the perception of faces includes some partly innate mechanisms and that face saliency during perception occurs early in development (Schultz et al. 2003; Rossion and de Heering 2015).

A network of neuro-anatomical areas is responsible for the visual and emotional perception of faces, with the fusiform face area (FFA) playing a key role. In fMRI studies, the FFA has repeatedly been identified as the most active region during face perception tasks

52 (Pierce et al. 2001). Consequently, the face-specificity hypothesis proposes that there exists 53 a distinct mechanism for face processing (Kanwisher and Yovel 2006; Rhodes et al. 2004). 54 The recognition of faces may therefore not only be functionally distinct from object recognition, but also anatomically segregated (Pitcher, Duchaine, and Walsh 2014). 
An alternative theory on the functional specificity of the FFA suggests that activity may be experience-related (Gauthier et al. 2000). The Domain-General theory suggests that the FFA is active in the processing of various stimuli, not just restricted to faces, as long as we can build experience with these objects. Since we continuously build experience with the faces we see around us, the FFA may just be primed to react strongly to faces due to this longpresent experience. Functional MRI studies have found evidence suggesting that face-related activity in the FFA is likely to become stronger with age due to a continuous increase of experience with faces (Nordt et al. 2018; Natu et al. 2016).

However, it has also been shown that the perception of faces and the recognition of both identity and emotion decline after a certain age (Ruffman et al. 2008; Mather 2016). Neural networks necessary for face processing also change with age (Mather 2016; Thomas et al. 2008). The superior temporal gyrus (STG), orbitofrontal cortex (OFC) and the amygdala have been suggested as part of the entire emotional brain (Baron-Cohen et al. 1999). Aging of these areas, particularly the STG (including the FFA and the pSTS) may affect the perception of faces during decline. In a diffusion tensor imaging (DTI) study by Thomas et al. it was found that the integrity of white matter tracts in the right FFA reduced with age (Thomas et al. 2008). The decline of neuro-anatomical regions which are involved in the perception and processing of social stimuli from the visual cortex to higher processes in the frontal regions, may therefore contribute to the decline of emotion recognition and selfreported empathy in aging individuals. The effect of age on face perception may therefore be an important factor to address while comparing the two hypotheses.

Perceptual categorization of faces is commonly found from a young age in human infants, and remains present throughout development. In a study by Rossion and de Heering (2015) it was shown that neurotypical infants as young as four months showed heightened neural responses elicited by the perception of faces. While infants from 3 years of age already respond stronger to faces in the FFA, reduced eye-contact and a diminished motivation to engage in social interaction (Pierce et al. 2001; Schultz et al. 2003; Moriuchi, Klin, and Jones 2017; Tanaka and Sung 2016) may result in individuals with ASC being 'face-inexperienced'. empathy and/or age we set up an electroencephalography (EEG) study using a paradigm 
87 periodical presentation of social stimuli within sequences of non-social stimuli and

88 quantifying a face-specific neural response based on the relative evoked potentials of the two

89 stimuli types. We then used an analysis called the Sequential Bayes Factors (SBF) to assess

90 the relation between face-related activity, cognitive empathy and age (Schönbrodt et al.

91 2015). SBF allows for the sequential computation of Bayes factors for the correlation

92 between two factors until a predefined level of evidence is attained. The SBF approach was

93 used to allow gradual and simultaneous participant-recruitment and analyses, with the

94 option to stop recruitment when the results produced clear converging evidence in support

95 of either the null or alternative hypotheses.

96 Results

97 We collected data from a group of largely neurotypical individuals (3 individuals reported a 98 diagnosis of ASC) as part of a larger ongoing EEG study. Participants $(\mathrm{N}=56)$ completed the 99 Autism Spectrum Quotient (AQ) and the 'Reading the Mind in the Eyes' Task. We measured 100 the face specific neural signature using EEG. Participants' ages ranged from 17 to 66 years 101 old (26 females and 30 males, mean age $=27.5 \mathrm{sd}=12.3$ ).

103 We recorded the EEG data using a BioSemi active two 64-electrode system. Participants wore 104 a biosemi cap with a 20-10 layout system, in which electrodes were inserted with saline-gel 105 filled plastic electrode holders. We used a fast serial visual presentation (FSVP), as used in 106 the study by Rossion and de Heering $(2015,9-10)$. The task exposes the viewer to a series of 107 images at a steady frequency (see methods and figure 8.A. (Rossion and de Heering 2015) for 108 details). The images used portray neutral scenes, such as objects and houses. These were 109 presented at a $6 \mathrm{~Hz}$ frequency, fading in and out using a sinusoidal contrast modulation. The 110 computer screen that we used during the task presentation has a $144 \mathrm{~Hz}$ refresh rate in order 111 to facilitate the rapid contrast modulation. 
1158 aperiodic trials in which face images were presented at random intervals. Each trial lasted 11614 seconds. With the use of an FSVP approach, we can isolate the exact frequency at which

117 stimulation is produced to measure the elicited response. As such it allows for both a 118 predictive and an objective analysis.

The periodic exposure to the stimulus allows the isolation of a specific voltage peak of 121 the signal at the determined frequency, known as: a steady state visual evoked potential 122 (SSVEP). We isolated the SSVEP and used it for the computation of the face-specific neural 123 signature for further analyses. Furthermore, we downsampled the signal to $1024 \mathrm{~Hz}$ during recording, as this provided enough temporal resolution to detect the SSVEP.

130 We plotted the association and sequential bayes of the separate relationships between the 131 face specific neural activity, cognitive empathy and age to assess the sampling effects, and to 132 evaluate the consistency across simulations.

133 The Relationship Between Cognitive Empathy and Face Specific Neural Activity

136 found decisive evidence for a lack of relationship between the face specific peak in the left 137 fusiform face area and RMET scores (Final Bayes Factor: 8.084) (Figure 1.). 


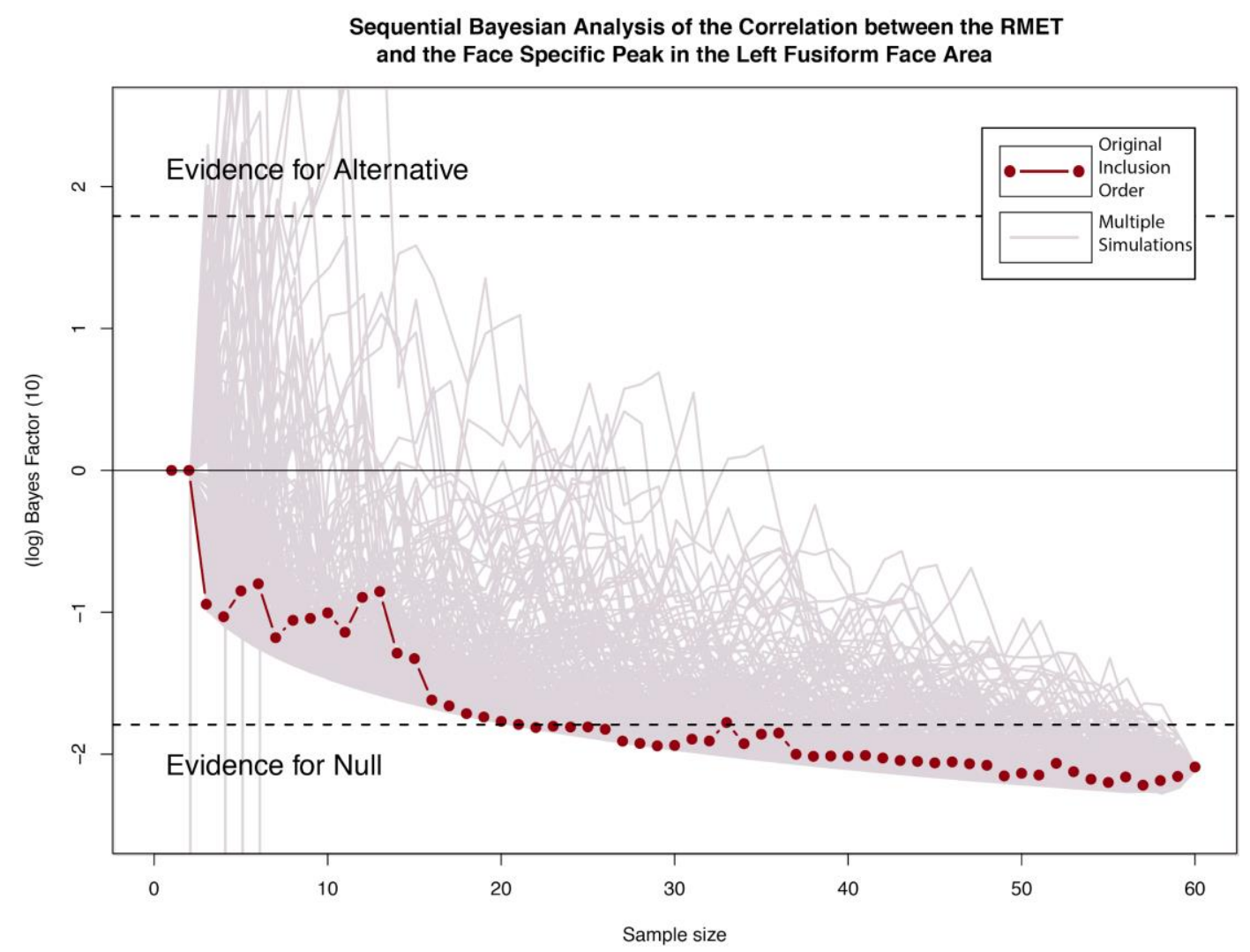

Figure 1. Sequential Bayesian Analysis of the Correlation between Accumulation of evidence throughout data collection for the correlation between RMET scores and the Face Specific Peak in the Left FFA (Final Bayes Factor: $\left.B F_{10}=8.084, B F_{01}=0.1234, \log _{10}\left(B F_{10}\right)=-2.090\right)$ The convergence of the multiple simulations indicates that there exists decisive evidence in favour of the null hypothesis.

148 We also found strong evidence for the lack of relationship between the face specific peak in 149 the right fusiform face area and RMET scores (Final Bayes Factor: 3.9024) (Figure 2.), 150 meaning there was no significant correlation between cognitive empathy and facial 151 perception as found in the fusiform face area in both hemispheres. As can be seen in both 152 Figures 1 and 2 the multiple simulations indicate that there was a high chance of false positives in lower sample sizes. This indicates that incorrect conclusions regarding the 
154 existence of a relationship could easily be drawn if an insufficient number of participants 155 were included in the analysis.

156

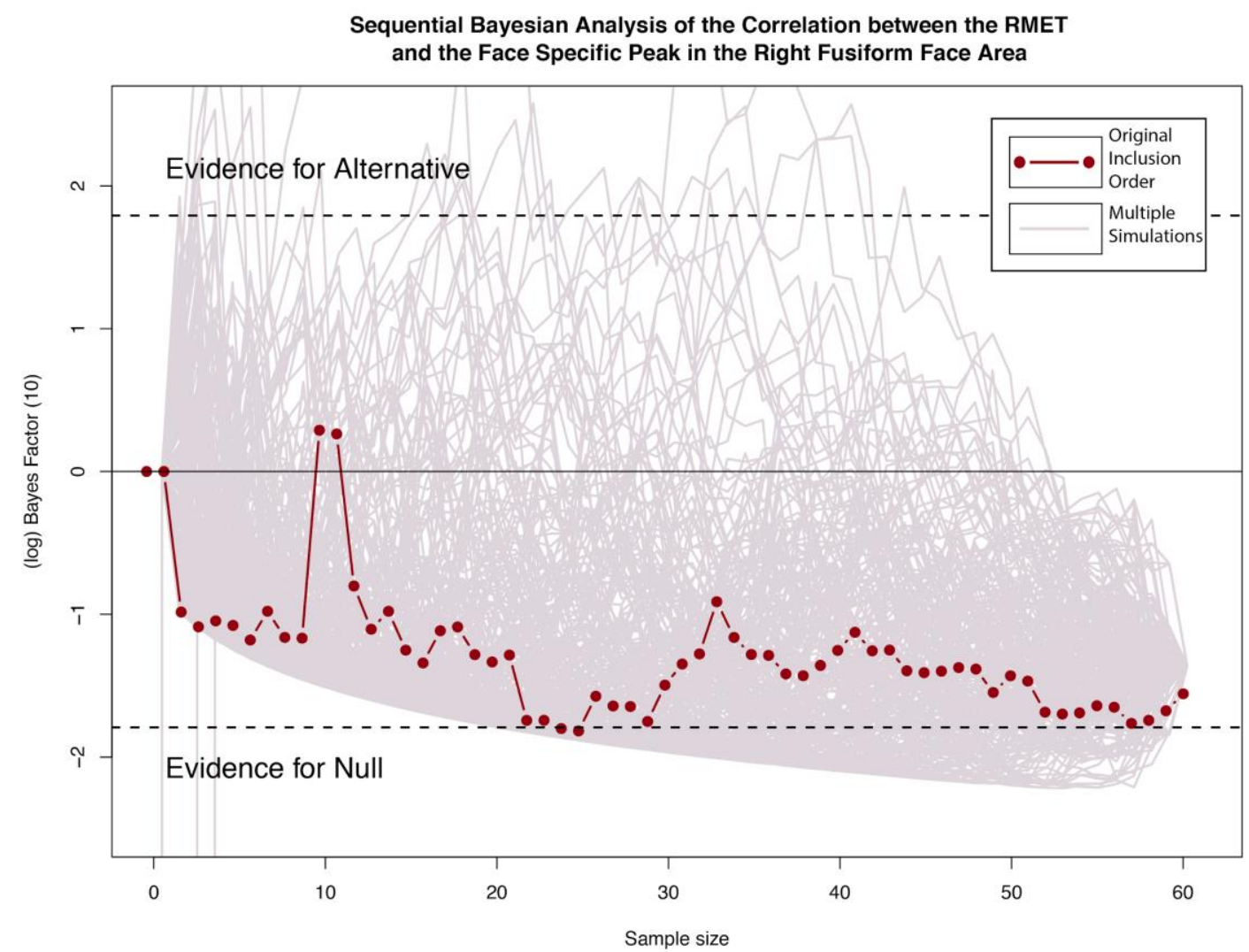

157

158

159

160

161

162

163

164

Figure 2. Sequential Bayesian Analysis of the Correlation between the RMEt and the Face Specific Peak in the Right Fusiform Face Area. Accumulation of evidence throughout data collection for the correlation between RMET scores and the Face Specific Peak in the Right FFA (Final Bayes Factor: $B F_{10}=3.9024, B F_{01}=0.256, \log _{10}\left(B F_{10}\right)=-$ 1.362) The convergence of the multiple simulations indicates that there exists strong evidence in favour of the null hypothesis.

165 The Relationship Between Cognitive Empathy and Age

166 Secondly, we plotted the association and sequential bayes of the relationship between age 167 and cognitive empathy. In this dataset there seems to be no significant relationship between 168 age and cognitive empathy. We found strong evidence for a lack of relationship (Final Bayes 
169 Factor $=4.970$ ), indicating that age may not influence cognitive empathy (Figure 3.). Again, a 170 substantial number of false positives could be found in the simulations, at a lower number of 171 sample sizes.

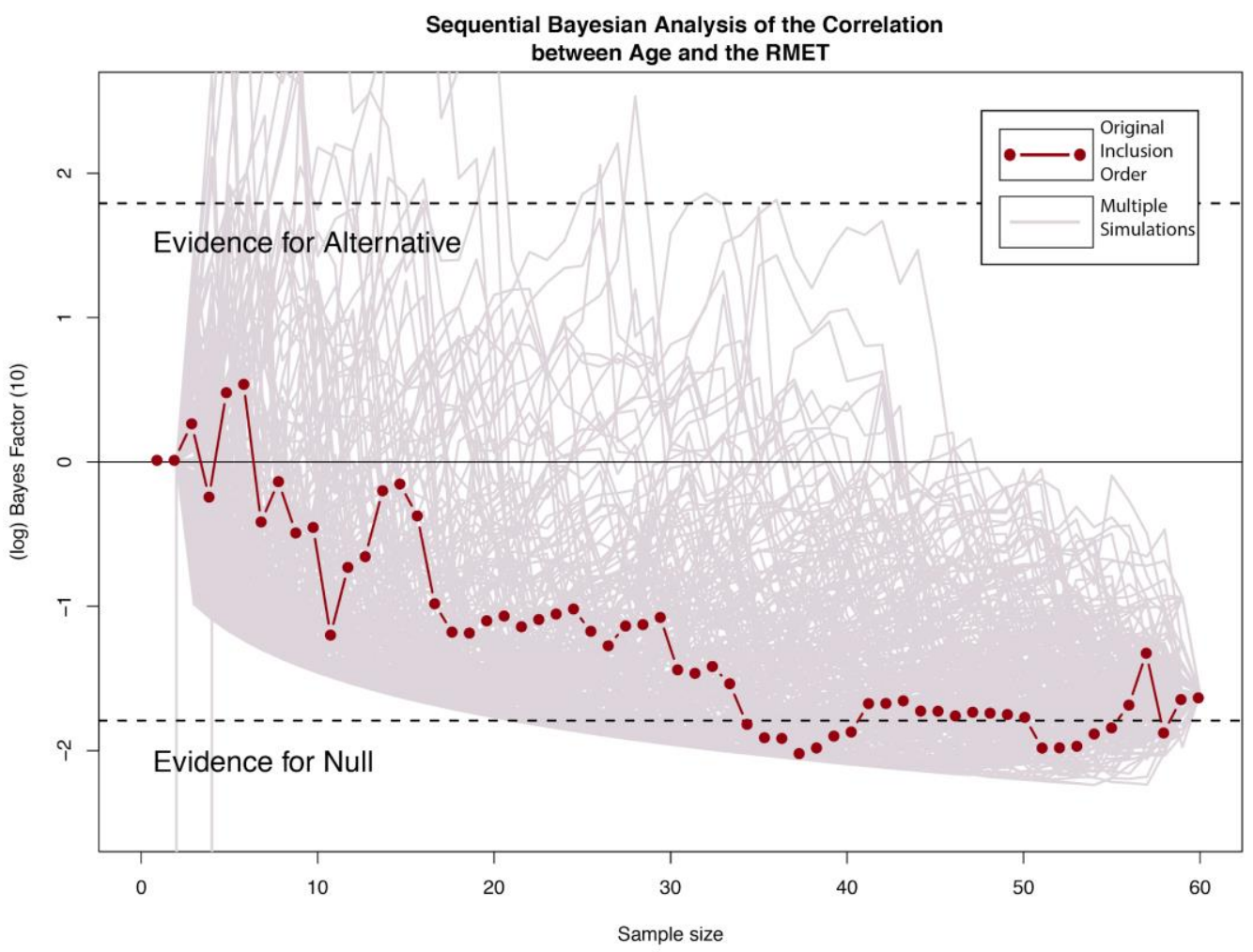

172

173

174

175

176

177

178

179

180 The Relationship Between Age and Face-Specific Neural Activity

181 Lastly, we plotted the association and sequential Bayes of the relationship between the face 182 183

\section{Figure 3. Sequential Bayesian Analysis of the Correlation between}

Age and the RMET. Accumulation of evidence throughout data collection for the correlation between Age and the RMET scores (Final Bayes Factor: $\left.B F_{10}=4.970, B F_{01}=0.2012174, \log _{10}\left(B F_{10}\right)=-1.603\right)$. The convergence of the multiple simulations indicates that there exists strong evidence in favour of the null hypothesis. specific neural activity and age. We found evidence for a lack of relationship between age and face-specific activity in both the fusiform face areas. Bayes Factors indicate no correlation 
184 between age and face specific peak frequency in neither the left (final BF $=5.390$, figure 4) nor the right (final $\mathrm{BF}=1.954$, figure 5) FFA. This indicates that age may not influence the activity in either the left or the right fusiform face area.

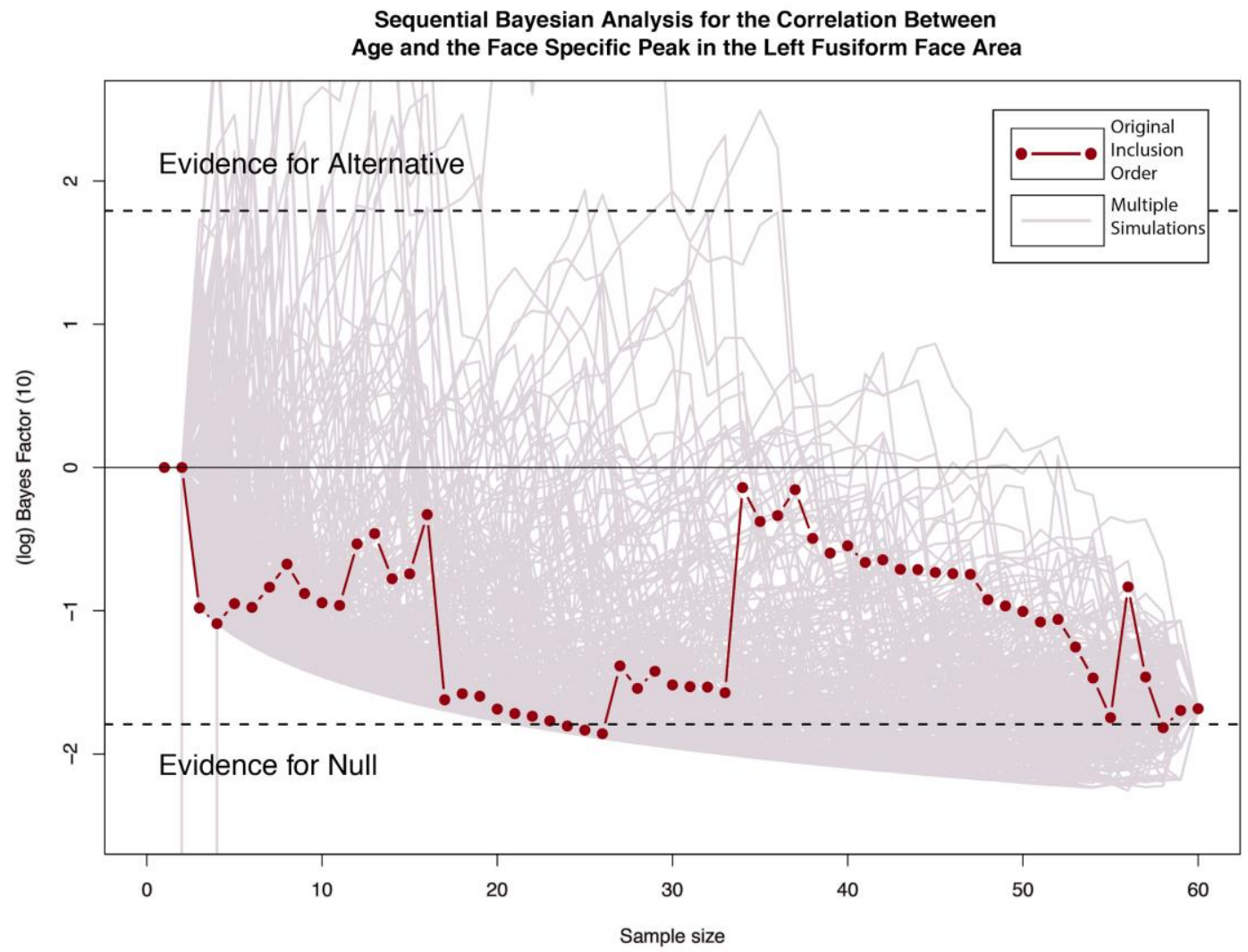

Figure 4.. Sequential Bayesian Analysis for the Correlation correlation between Age and the Face Specific Peak in the Left FFA $\left(\left(B F_{10}=5.390, B F_{O I}=0.1856731, \log _{10}\left(B F_{10}\right)=-1.684\right)\right.$. The convergence of the multiple simulations indicates that there exists strong evidence in favour of the null hypothesis. 


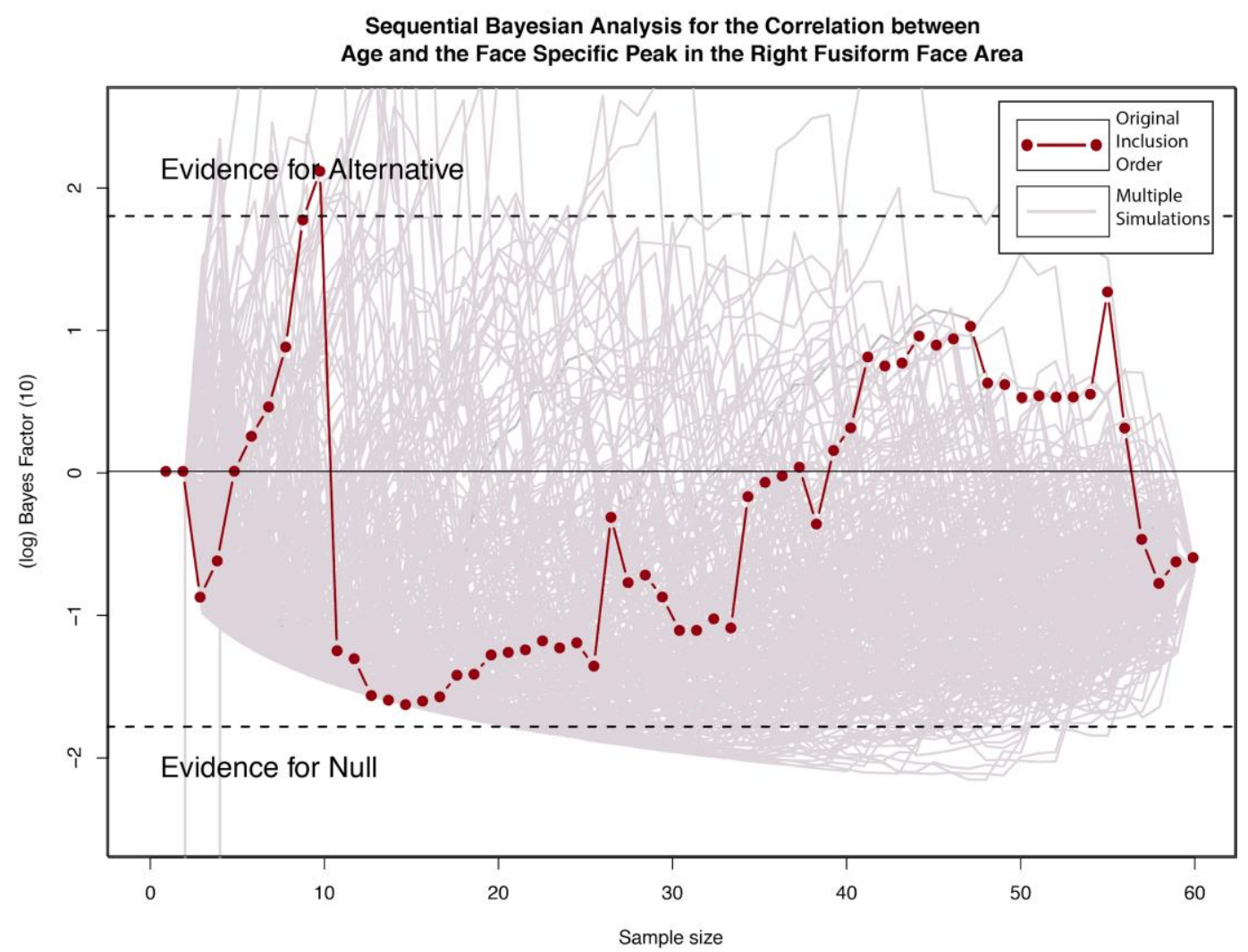

Figure 5.. Sequential Bayesian Analysis for the Correlation Between

Age and the Face Specific Peak in the Right Fusiform Face Area.

205 In all of the plotted simulations a high number of false positives could be found, although null hypothesis.

\section{7 assessment of sampling effects.}

\section{Discussion}

209 The present study aimed to test if there is a correlation between a face-specific neural 210 signature and cognitive empathy in a typical participant group. With the use of a validated 
211 behavioural measure of cognitive empathy, the 'Reading the Mind in the Eyes' Test, a

212 significant relation between the two could have established the face-specific neural signature

213 as a potential biological neuromarker of cognitive empathy (Simon Baron-Cohen et al. 2001).

214 Our Sequential Bayesian Analysis indicated that with our participant group there was strong

215 evidence against such an association.

216

217 There may be several reasons for the lack of relationship between the RMET and the face-

218 specific neural signature in this dataset. Firstly, the face-specific neural signature used in this

219 study, the steady state visually evoked potential during face presentation in the FFA, is a low 220 level aspect of visual perception of faces (Rossion and Boremanse 2011). Therefore, facespecific neural signature may be too low-level a process to have an immediate relationship with a higher-level concept such as that of cognitive empathy.

223

224

It is possible that at this low-level stage of perception no deviations exist, but that instead an altered integration of perceived stimuli further along the visual processing stream influences cognitive empathy. The current FSVP task concerns purely the classification of faces versus neutral images. No emotionally salient aspects or features were perceived that required higher levels of integration, such as emotional recognition, gaze following or holistic faceprocessing. While there may be individual differences at the low-level perception of faces vs non-faces, these may not have a direct relationship with the level of cognitive empathy. This explanation of our results aligns with the Weak Central Coherence theory (Happé 1996; López et al. 2004), which posits that fundamental perception is intact or even heightened for simple features in individuals with ASC, yet that the integration of these perceptions at a higher processing level, for example during emotion processing, is affected. As such, there may be no altered sensory perception affecting cognitive empathy, but an altered integration of sensory input. As the RMET requires higher-level processing (Fernández-Abascal et al. 2013; Preston et al. 2007; Moor et al. 2012), it is likely that the local processing bias has no direct link with an individual's performance on this task, but rather the inability to integrate the separately perceived social stimuli .

Furthermore, we studied a participant group of mostly neurotypical individuals. 
242 dysfunction of cognitive empathy was set, it may be the case that a relationship between

243 facial perception and cognitive empathy is only noticeable at pathological levels. The lack of

244 individuals with an actual ASC diagnosis in our participant sample, may have prevented us

245 from finding a significant relationship between face perception and cognitive empathy, as

246 cognitive empathy has been found to be significantly lowered in individuals with ASC

247 (Peñuelas-Calvo et al. 2019; Schultz et al. 2003; Schultz 2005). Therefore we suggest that

248 future studies may focus their attention on the possible relationship between holistic face-

249 processing and cognitive empathy, and include a higher number of participants with an ASC

250 diagnosis.

251

According to the experience-hypothesis, one would expect a better ability to judge

252

emotion and facial expression with older age. Aging has been proven to lead to an increase in cognitive empathy up to a certain age (Thomas et al. 2008; Ruffman et al. 2008; Nordt et al. 2018); with older age cognitive decline and thereby a decrease of cognitive empathy is common, as neural capacity and executive functioning decrease. In our sample we found evidence for the lack of a relationship between age and cognitive empathy, as well as age and the face-specific neural signature. The evidence for the lack of a relationship seems to contradict the experience hypothesis of the fusiform face area. The distribution of age in the current sample, however, may have prevented us from finding this relationship (Figure 6.B.). Since the distribution of age in our sample is skewed, this evidence may be futile. Future studies assessing this hypothesis therefore need to consider age distribution when assessing the effect of age on neural signatures and cognitive empathy.

This study was designed around the premise that steady state visual evoked potentials (SSVEP) could be triggered in the FFA, through a fast serial visual presentation (FSVP) of imagery, as was similarly done in a previous study conducted by Rossion et al. (2015). The detected SSVEP indicated that there is a stronger response in the FFA towards faces rather than objects (Figure 8.B.). This result suggests that the methodology is valid, and that the FFA shows a heightened response to face-imagery, as has been found in previous studies (Kanwisher and Yovel 2006; Rossion and Boremanse 2011). However, while this

270 finding is in line with the face-specific hypothesis, it is not sufficient to disprove the experience-related hypothesis (Gauthier et al. 2000; Rhodes et al. 2004). 
Therefore we have shown the functionality of an EEG paradigm based on the SSVEP, 273 the efficiency of the analysis, and its ability to eliminate possible participant recruitment

274 order effects. The sequential Bayesian analysis has facilitated analysis during the on-going 275 recruitment of participants, by allowing us to bypass the process of setting a predefined 276 inclusion number of participants before analysis could take place. Thus, the use of an SBF can 277 allow for flexibility in sample-planning and facilitate this process when participants are 278 harder to recruit, for example in rare clinical samples. In the case of unclarity in terms of 279 existing relationships, it is possible to include more data-points in an unbiased manner while 280 increasing the accuracy of the effect size (Schönbrodt et al. 2015). Furthermore, the SBF makes for an intuitive tool to account for sampling order. Especially in lower sample sizes

282 the sequential Bayesian simulation showed that there are likely quite a few false positives in 283 our sample. This could be a result of order-effects in smaller sample sizes. Thus, without the 284 SBF analysis it would be possible to make premature conclusions on existing relationships, 285 whereas with the use of the SBF we could ensure that there was no evidentiary support for 286 the relationships in question.

In summary, by using sequential Bayesian analysis we found no evidence for an association between cognitive empathy, facial visual perception or age. However, we have highlighted the usefulness of SBF to determine appropriate sample size and stopping criteria. We acknowledge that an association may exist but may be precluded by the current sample distribution. Future studies should consider adopting a similar bayesian framework for robust hypothesis evaluation.

294 Materials and Methods

297 We visualized the distribution of data points within our sample using Raincloud 298 Plots. We created these with the use of code developed by Micah Allen (Allen et al. 299 2019). While the RMET scores (Figure 6.A) show a rather normal distribution, the 300 Age and AQ scores (Figure 6.B and 6.C respectively) show a fairly skewed data point 
301 distribution. Further information on the correlation between these measures can be

302 found in the Supplementary Materials.

303

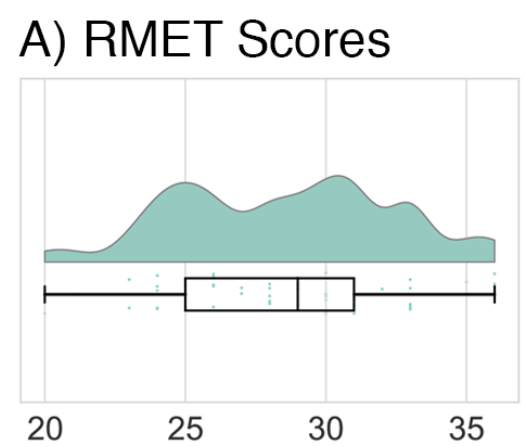

B) Age

304

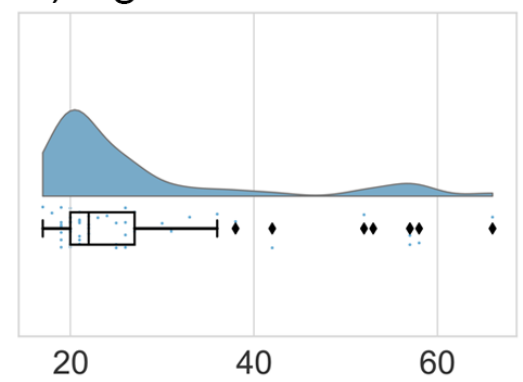

C) AQ Scores

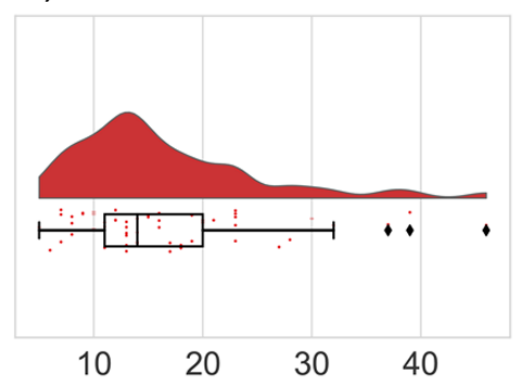

305 Figure 6. Distribution of RMET, AQ and Ages of the included participant group $(n=56)$ A)

306 RMET scores B) Age C) Autism Quotient Scores

307

308 The Reading the Mind in the Eyes Task

309

310 We measured cognitive empathy by means of the Reading the Mind in the Eyes Test (RMET)

311 (See Figure 7.B). This test has been validated as an appropriate behavioural measure of

312 cognitive empathy (Fernández-Abascal et al. 2013). It measures the extent to which

313 individuals can attribute and recognise people's facial expressions solely via a cropped facial

314 area around the eye (Simon Baron-Cohen et al. 2001). The RMET has been found to have good

315 reliability, as individuals with ASC consistently score lower on the task (Fernández-Abascal

316 et al. 2013; Baron-Cohen et al. 1997). As such the RMET has often been used in various types

317 of research studying the mechanisms behind cognitive empathy (Warrier et al. 2018; Baron-

318 Cohen et al. 2015; Greenberg et al. 2018). 


\section{A) Fast serial visual presentation task}

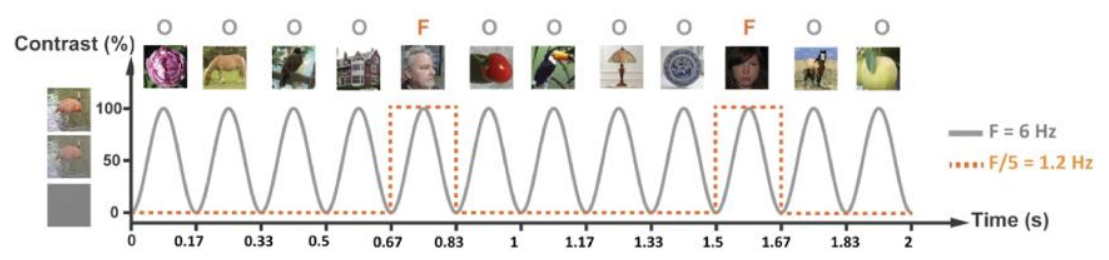

B) Reading the mind in the eye task
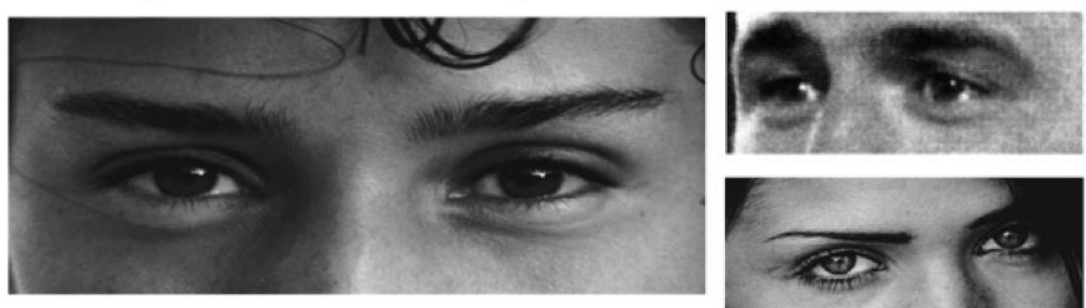

playful

comforting

$\mathrm{O}$ irritated

$\mathrm{O}$ bored

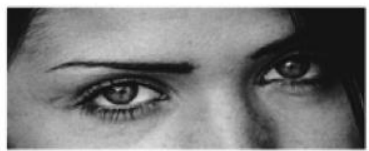

C) Spectral density

D) FOOOF Model
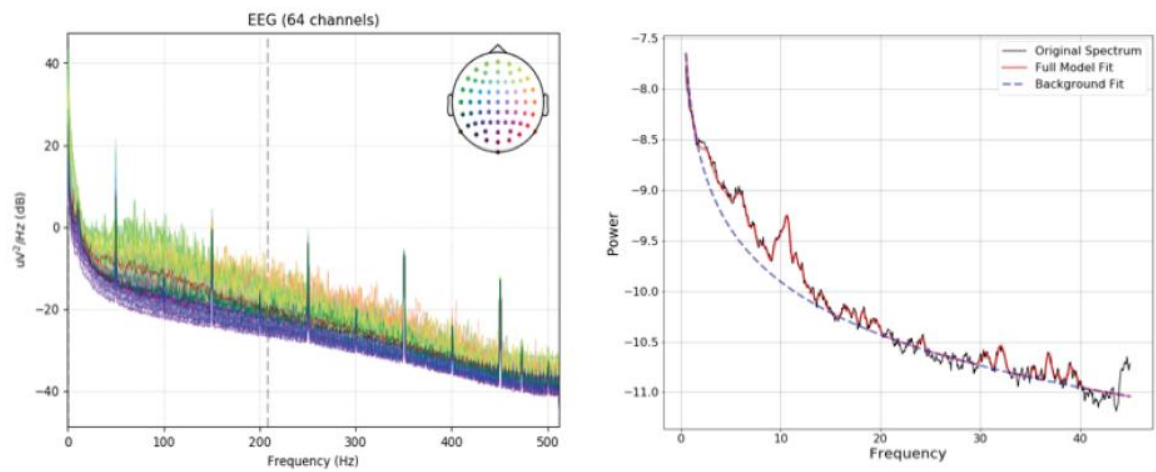

Figure 7. A Fast Serial Visual Presentation Task. B. The Reading the Mind in the Eyes Task. C. Spectral Density. D. FO00F Model

327 We preprocessed and analysed each acquired EEG dataset using MNE software in Python

3283.6 (available at https://mne.tools/dev/install/index.html and 
https://www.python.org/downloads/release/python-366/)(Gramfort et al. 2013). The raw

data was separated in 14 second epochs which were assessed for quality. Epoch quality was

331 assessed both manually and using AutoReject: Bad channels were interpolated where possible, using the Random Sample Consensus (Ransac) function of AutoReject (Jas et al. 2017; Bigdely-Shamlo et al. 2015). The Ransac algorithm determines the data quality of

334 each channel and, in case of bad quality, repairs these. Thereafter, we determined the quality of each epoch-object and cleaned the dataset of bad epochs. If less than 11 out of 16 epochs remain from a dataset (68.75\%), we removed it from analysis. At this stage 8 datasets were excluded due to noisy epochs. The raw data was filtered (0.5 - 15), demeaned and underwent linear detrending. We performed a Fast Fourier Transform (FFT) to construct a data structure for the SSVEP analysis, determining the signal to noise (SNR) ratio during the frequencies of our interest $(6 \mathrm{~Hz}$ and $1.2 \mathrm{~Hz})$. Jan Freyberg developed the code that we used in order to do this (https://github.com/ianfreyberg/face-perceptionssvep) We computed the SNR by comparing the signal level during the stimulus to the signal level without the stimulus.

\section{Controlling for an Aperiodic Signal}

345 EEG signals are inherently noisy, and the amplitude of this noise changes with frequency. A

346 large component of this signal is proportional to the inverse of the frequency $(1 / f)$, and is 347 therefore often called the aperiodic component of neural power spectra. Given that there are 348 individual differences in the parameters of this noise (see Voytek et al, 2015), we aimed to 349 subtract this noise signal from each participant's EEG spectrum. We used the algorithm Fit 350 Oscillations Of One Over F (FOOOF, (Haller et al. 2018). The aperiodic fit is estimated using 351 linear regression in log-space. This estimate is then subtracted from the power spectrum. The 352 algorithm then iteratively fits gaussians to peaks in the remaining power spectrum until a 353 threshold is reached (Figure 7.D.).

355 Visualization and Comparison of the face-specific and baseline neural signature

356 We used peaks identified by the FOOOF algorithm as estimates of neural oscillation at the 357 stimulation frequencies $(1.2$ and $6 \mathrm{~Hz})$. In Figure 8.A. visualization of the average peak 
359 of the neural activity during the frequencies of interest shows increased high activity in the occipital cortex during $6 \mathrm{~Hz}$, activity was similar during periodic and aperiodic trials. In the 1.2 Hz frequency overall activity was lower. During Periodic trials increased activity could be seen in the occipital cortex and in the left and right FFA, as well as in the T8 and TP8 electrodes; the posterior superior temporal sulcus of the right hemisphere.

A)

$1.2 \mathrm{~Hz}$

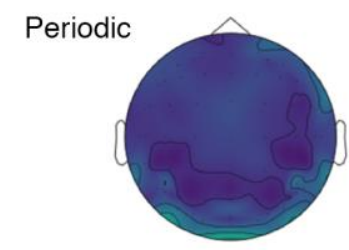

Aperiodic

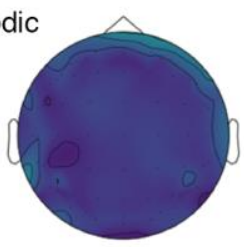

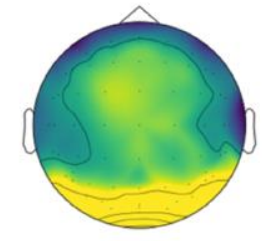

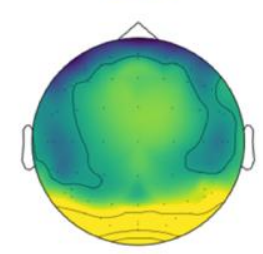

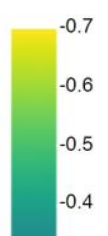

올

B)

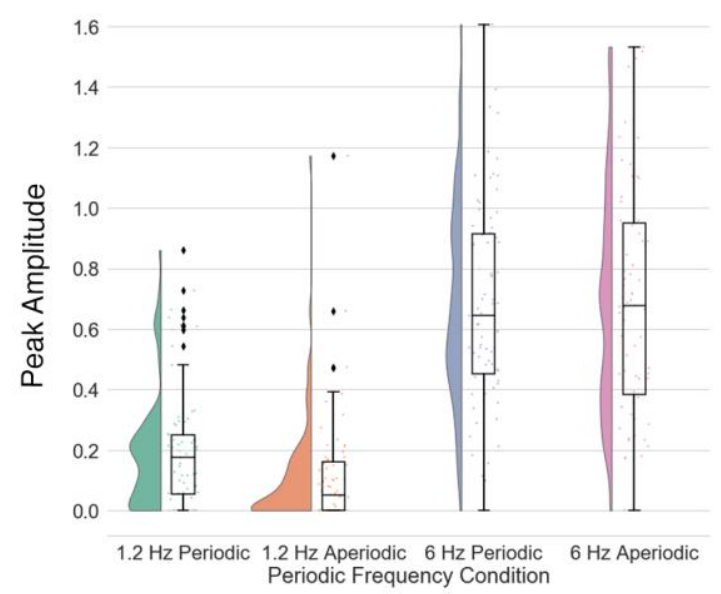

364

365

366

367

368

369

370

371

372

373

374

375

376

377

378

379

Figure 8 . A) Topo Maps of the peak amplitude per periodicity and frequency. B) RainCloud Distribution of the average peak amplitude per periodicity and frequency in the right FFA periodic baseline peak (M: 0.742659, SD: 0.331077), aperiodic baseline peak (M: 0.551727, SD: 0.325041) periodic face-specific peak (M: 0.207213, SD: 0.205435) aperiodic face-specific peak (M: 0.118524, SD 0.192918)

The distribution of the average peak amplitude in the right FFA for each participant is visualized per condition in figure 8.B. using a Rain-cloud plot method (as translated to Python https://github.com/pog87/PtitPrince) (Allen et al. 2019). An independent t-test of the baseline peak amplitudes over periodic (M: 0.74, SD: 0.33) and aperiodic trials (M: 0.55, SD: $0.32)$ differed significantly $(\mathrm{t}=4.00, \mathrm{p}=.0001)$. The face-related peak amplitudes have a lower peak in general due to the lower presentation frequency. The plot shows that the face-specific periodic peak distribution (M: 0.21 , SD: 0.21$)$ is significantly higher $(t=3.01, p=0.004)$ than 
380 the aperiodic peak distribution (M: 0.12, SD 0.19). Therefore, looking at the peak amplitude 381 of the periodic face-frequency, it can be concluded that there is a clear SSVEP in response to

382 face-images as compared to neutral images.

383

384 Sequential Bayesian Factor

385 The evidence for or against the null hypothesis is determined through the calculation of the 386 Bayesian Factor (BF). The BF is the ratio of one hypothesis being more likely than the other.

387 The $\mathrm{BF}_{10}$ is the ratio of the likelihood of the alternative hypothesis (H1) versus the likelihood 388 of the null hypothesis ( $\mathrm{H} 0)$. We chose a threshold of $\left|\log \left(\mathrm{BF}_{10}\right)\right|>1.8$, reflecting either 389 hypothesis being 63x higher than the other. According to Kass and Raftery (1995) this 390 indicates 'strong' evidence. Sequential bayes factor methods allow assessment of the 391 accumulation of evidence using random sampling. Here, instead of simply evaluating the 392 Bayes Factor with each new participant, after every new data point, we simulated the 393 experiment with a new, random order of participants. This facilitates the visualisation of the 394 trend in evidence over the course of the experiment without confounding order effects.

396 Contributions

\begin{tabular}{|c|c|c|c|c|c|}
\hline 397 & J.D. Kist: & 409 & Methodology & 421 & Crockford, Elke de \\
\hline 398 & Conceptualization & 410 & Validation & 422 & Jonge: \\
\hline 399 & Methodology & 411 & Investigation & 423 & Investigation \\
\hline 400 & Software & 412 & Resources & 424 & \\
\hline 401 & Formal analysis & 413 & Data curation & 425 & Jan Freyberg: \\
\hline 402 & Investigation & 414 & Writing & 426 & Conceptualization \\
\hline 403 & Data curation & 415 & Supervision & 427 & Methodology \\
\hline 404 & Writing & 416 & Project administration & 428 & Software \\
\hline 405 & Visualization & 417 & Funding & 429 & Validation \\
\hline 406 & & 418 & & 430 & Investigation \\
\hline 407 & R.A.I. Bethlehem: & 419 & Barnaby Stonier, & 431 & Resources \\
\hline 408 & Conceptualization & 420 & Olivier Sluijters, Sarah & 432 & Writing (review) \\
\hline
\end{tabular}




\begin{tabular}{|c|c|c|c|c|c|}
\hline 433 & Visualization & 440 & Funding & 447 & Validation \\
\hline 434 & Supervision & 441 & Supervision & 448 & Investigation \\
\hline 435 & Project administration & 442 & & 449 & Resources \\
\hline 436 & & 443 & O.E. Parsons: & 450 & Writing (review) \\
\hline 437 & Simon Baron-Cohen: & 444 & Conceptualization & 451 & Visualization \\
\hline 438 & Conceptualization & 445 & Methodology & 452 & Supervision \\
\hline 439 & Resources & 446 & Software & 453 & Project administı \\
\hline
\end{tabular}

455 Competing interests

456 There are no competing interests.

457

458 Bibliography

459 Allen, Micah, Davide Poggiali, Kirstie Whitaker, Tom Rhys Marshall, and Rogier A. Kievit.

460 2019. "Raincloud Plots: A Multi-Platform Tool for Robust Data Visualization." Wellcome

$461 \quad$ Open Research 4 (April): 63.

462 American Psychiatric Association. 2013. Diagnostic and Statistical Manual of Mental

463 Disorders. Fifth Edit. Alrington, VA.

464 Baron-Cohen, S. 2011. "Zero Degrees of Empathy: A New Understanding of Cruelty and

465 Kindness." Allen Lane, London.

466 Baron-Cohen, Simon, Sally Wheelwright, Jacqueline Hill, Yogini Raste, and Ian Plumb. 2001.

467 "The 'Reading the Mind in the Eyes' Test Revised Version: A Study with Normal Adults,

468 and Adults with Asperger Syndrome or High-Functioning Autism." Journal of Child

469 Psychology and Psychiatry, and Allied Disciplines 42 (2): 241-51.

470 Baron-Cohen, Simon, Sally Wheelwright, Jolliffe, and Therese. 1997. "Is There a' Language of

471 the Eyes'? Evidence from Normal Adults, and Adults with Autism or Asperger

472 Syndrome." Visual Cognition 4 (3): 311-31.

473 Baron-Cohen, S., H. A. Ring, S. Wheelwright, E. T. Bullmore, M. J. Brammer, A. Simmons, and

474 S. C. Williams. 1999. "Social Intelligence in the Normal and Autistic Brain: An fMRI 
Study." The European Journal of Neuroscience 11 (6): 1891-98.

476

477

478

479

480

481

482

483

484

485

486

487

488

489

490

491

492

493

494

495

496

497

498

499

500

501

502

503

504

505

Bigdely-Shamlo, Nima, Tim Mullen, Christian Kothe, Kyung-Min Su, and Kay A. Robbins. 2015. “The PREP Pipeline: Standardized Preprocessing for Large-Scale EEG Analysis.” Frontiers in Neuroinformatics 9 (June): 16.

Dellapiazza, Florine. 2018. "Links between Sensory Processing, Adaptive Behaviours, and Attention in Children with Autism Spectrum Disorder: A Systematic Review." Psychiatry Research 270 (August): 78-88.

Fernández-Abascal, Enrique G., Rosario Cabello, Pablo Fernández-Berrocal, and Simon Baron-Cohen. 2013. "Test-Retest Reliability of the 'Reading the Mind in the Eyes' Test: A One-Year Follow-up Study." Molecular Autism 4 (1): 33.

Gauthier, I., P. Skudlarski, J. C. Gore, and A. W. Anderson. 2000. “Expertise for Cars and Birds Recruits Brain Areas Involved in Face Recognition." Nature Neuroscience 3 (2): 191-97.

Gramfort, Alexandre, Martin Luessi, Eric Larson, Denis A. Engemann, Daniel Strohmeier, Christian Brodbeck, Roman Goj, et al. 2013. "MEG and EEG Data Analysis with MNEPython." Frontiers in Neuroscience 7 (December): 267.

Greenberg, D. M., V. Warrier, and C. Allison. 2018. “Testing the Empathizing-Systemizing Theory of Sex Differences and the Extreme Male Brain Theory of Autism in Half a Million People." Proceedings of the. https://www.pnas.org/content/115/48/12152.short.

Haller, Matar, Thomas Donoghue, Erik Peterson, Paroma Varma, Priyadarshini Sebastian, Richard Gao, Torben Noto, Robert T. Knight, Avgusta Shestyuk, and Bradley Voytek. 2018. "Parameterizing Neural Power Spectra." Neuroscience. bioRxiv. https://doi.org/10.1101/299859.

Happé, F. G. 1996. "Studying Weak Central Coherence at Low Levels: Children with Autism Do Not Succumb to Visual Illusions. A Research Note." Journal of Child Psychology and Psychiatry, and Allied Disciplines 37 (7): 873-77.

Jas, Mainak, Denis A. Engemann, Yousra Bekhti, Federico Raimondo, and Alexandre Gramfort. 2017. “Autoreject: Automated Artifact Rejection for MEG and EEG Data.” NeuroImage 159 (October): 417-29.

Jones, Alice P., Francesca G. E. Happé, Francesca Gilbert, Stephanie Burnett, and Essi Viding. 2010. “Feeling, Caring, Knowing: Different Types of Empathy Deficit in Boys with 

and Psychiatry, and Allied Disciplines 51 (11): 1188-97.

508 Kanwisher, Nancy, and Galit Yovel. 2006. "The Fusiform Face Area: A Cortical Region Specialized for the Perception of Faces." Philosophical Transactions of the Royal Society

511 Kass, Robert E., and Adrian E. Raftery. 1995. “Bayes Factors.” Journal of the American

$512 \quad$ Statistical Association 90 (430): 773-95.

513 López, Beatriz, Nick Donnelly, Julie Hadwin, and Susan Leekam. 2004. "Face Processing in

$514 \quad$ High-functioning Adolescents with Autism: Evidence for Weak Central Coherence."

$515 \quad$ Visual Cognition 11 (6): 673-88.

516 Mather, Mara. 2016. "The Affective Neuroscience of Aging." Annual Review of Psychology 67: $517 \quad 213-38$.

518 Moor, Bregtje Gunther, Zdena A. Op de Macks, Berna Güroglu, Serge A. R. B. Rombouts,

519 Maurits W. Van der Molen, and Eveline A. Crone. 2012. "Neurodevelopmental Changes

520 of Reading the Mind in the Eyes." Social Cognitive and Affective Neuroscience 7 (1): 4452152.

522 Moriuchi, Jennifer M., Ami Klin, and Warren Jones. 2017. "Mechanisms of Diminished Attention to Eyes in Autism." The American Journal of Psychiatry 174 (1): 26-35.

524 Natu, Vaidehi S., Michael A. Barnett, Jake Hartley, Jesse Gomez, Anthony Stigliani, and 525 Kalanit Grill-Spector. 2016. “Development of Neural Sensitivity to Face Identity Correlates with Perceptual Discriminability." The Journal of Neuroscience: The Official Journal of the Society for Neuroscience 36 (42): 10893-907.

Nordt, Marisa, Kilian Semmelmann, Erhan Genç, and Sarah Weigelt. 2018. “Age-Related Increase of Image-Invariance in the Fusiform Face Area." Developmental Cognitive Neuroscience 31 (June): 46-57.

Peñuelas-Calvo, Inmaculada, Aditya Sareen, Julia Sevilla-Llewellyn-Jones, and Pablo

535 Pierce, K., R-A Müller, J. Ambrose, G. Allen, and E. Courchesne. 2001. "Face Processing 536 Occurs Outside the Fusiform `face Area' in Autism: Evidence from Functional MRI.” 
Brain: A Journal of Neurology 124 (10): 2059-73.

538 Pitcher, David, Bradley Duchaine, and Vincent Walsh. 2014. “Combined TMS and FMRI

539 Reveal Dissociable Cortical Pathways for Dynamic and Static Face Perception." Current

540 Biology: CB 24 (17): 2066-70.

541 Preston, Stephanie D., Antoine Bechara, Hanna Damasio, Thomas J. Grabowski, R. Brent

542 Stansfield, Sonya Mehta, and Antonio R. Damasio. 2007. "The Neural Substrates of

543 Cognitive Empathy." Social Neuroscience 2 (3-4): 254-75.

544 Rhodes, Gillian, Graham Byatt, Patricia T. Michie, and Aina Puce. 2004. "Is the Fusiform Face

545 Area Specialized for Faces, Individuation, or Expert Individuation?" Journal of Cognitive

$546 \quad$ Neuroscience 16 (2): 189-203.

547 Robertson, Caroline E., and Simon Baron-Cohen. 2017. "Sensory Perception in Autism."

$548 \quad$ Nature Reviews. Neuroscience 18 (11): 671-84.

549 Rossion, Bruno, and Adriano Boremanse. 2011. "Robust Sensitivity to Facial Identity in the

550 Right Human Occipito-Temporal Cortex as Revealed by Steady-State Visual-Evoked

551 Potentials." Journal of Vision 11 (2). https://doi.org/10.1167/11.2.16.

552 Rossion, Bruno, and Adelaide de Heering. 2015. "Rapid Categorization of Natural Face

553 Images in the Infant Right Hemisphere." eLife 4 (e06564.): 1-14.

554 Rueda, P., P. Fernández-Berrocal, and S. Baron-Cohen. 2015. “Dissociation between

555 Cognitive and Affective Empathy in Youth with Asperger Syndrome." The European

$556 \quad$ Journal of Developmental Psychology 12 (1): 85-98.

557 Ruffman, Ted, Julie D. Henry, Vicki Livingstone, and Louise H. Phillips. 2008. “A Meta-

558 Analytic Review of Emotion Recognition and Aging: Implications for

559

560 Neuropsychological Models of Aging." Neuroscience and Biobehavioral Reviews 32 (4): 863-81.

Schönbrodt, Felix D., Eric-Jan Wagenmakers, Michael Zehetleitner, and Marco Perugini. 2015. “Sequential Hypothesis Testing With Bayes Factors: E Ciently Testing Mean Di Erences." Psychological Methods 22 (2): 322-39.

Schultz, Robert T. 2005. "Developmental Deficits in Social Perception in Autism: The Role of 565 the Amygdala and Fusiform Face Area." International Journal of Developmental 
568 Schultz, Robert T., David J. Grelotti, Ami Klin, Jamie Kleinman, Christiaan Van Der Gaag, René Marois, and Pawel Skudlarski. 2003. "The Role of the Fusiform Face Area in Social Cognition: Implications for the Pathobiology of Autism." Philosophical Transactions of the Royal Society of London. Series B, Biological Sciences 358 (1430): 415-27.

Tanaka, James W., and Andrew Sung. 2016. “The ‘Eye Avoidance’ Hypothesis of Autism Face Processing." Journal of Autism and Developmental Disorders 46 (5): 1538-52.

574 Thomas, Cibu, Linda Moya, Galia Avidan, Kate Humphreys, Kwan Jin Jung, Mary A. Peterson, 575 and Marlene Behrmann. 2008. "Reduction in White Matter Connectivity, Revealed by Diffusion Tensor Imaging, May Account for Age-Related Changes in Face Perception."

578 Thye, Melissa D., Haley M. Bednarz, Abbey J. Herringshaw, Emma B. Sartin, and Rajesh K. Kana. 2018. "The Impact of Atypical Sensory Processing on Social Impairments in Autism Spectrum Disorder." Developmental Cognitive Neuroscience 29 (January): 15167.

Walter, Henrik, Birgit Abler, Angela Ciaramidaro, and Susanne Erk. 2005. "Motivating Forces of Human Actions. Neuroimaging Reward and Social Interaction." Brain Research Bulletin 67 (5): 368-81. among Individuals with Autistic Traits." Cognition 171 (February): 1-9. 


\section{Supplementary Materials}

589 An exploratory correlation between the face-specific neural signature in the left and right

590 fusiform face area, the Autism Spectrum Quotient, the RMET scores and age was performed.

591 In Figure 9.A we performed a Pearson Correlation with all the different variables, which

592 can be seen in the heatmap. In Figure 9.B a heatmap can be seen in which a Spearman

593 Correlation was performed.

594

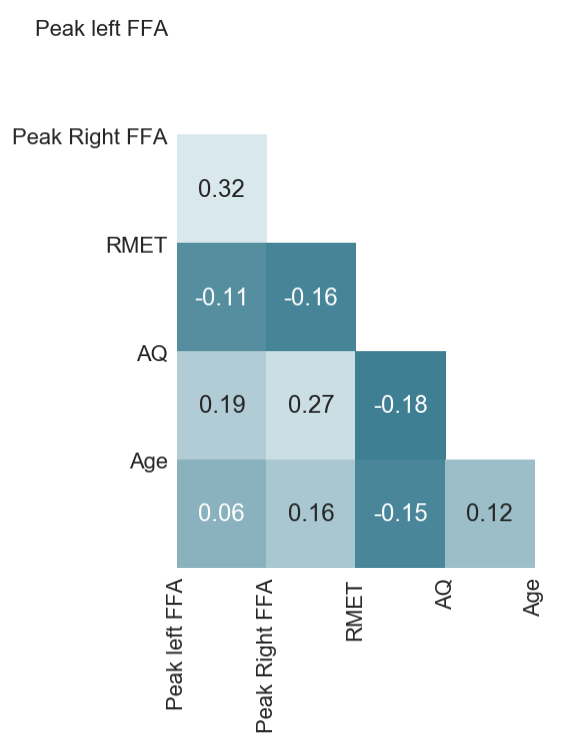

A)

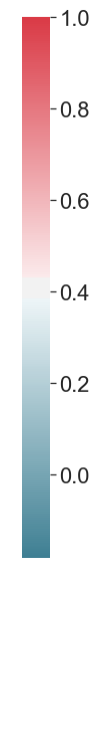

B)

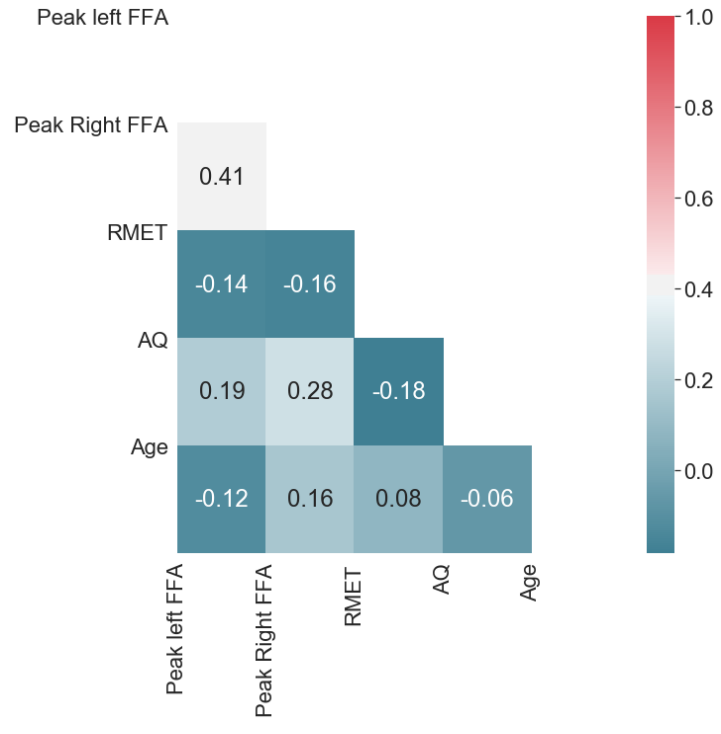


606 We visualized the different correlations by means of a scatter plot. The different

607 relationships can be seen in Figure 10.

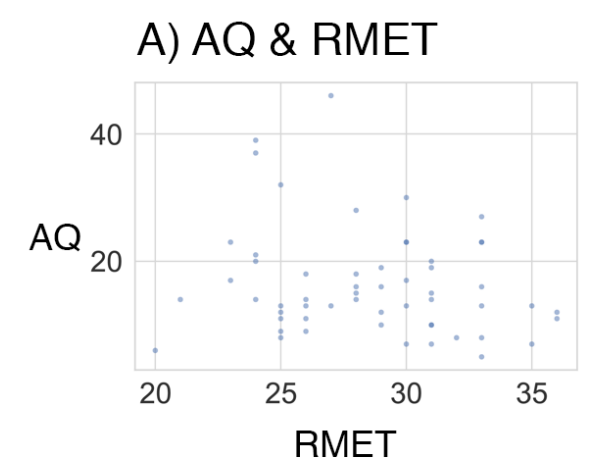

B) $A Q \&$ Age

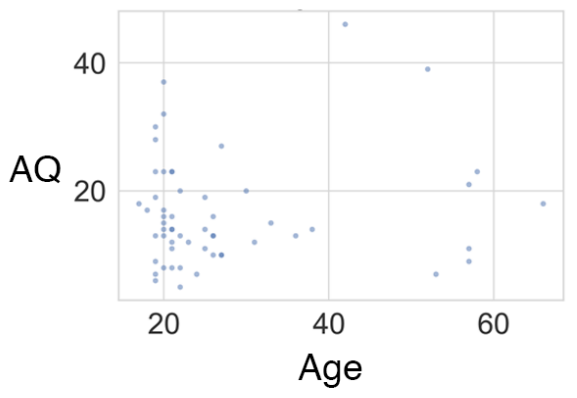

C) RMET \& Age

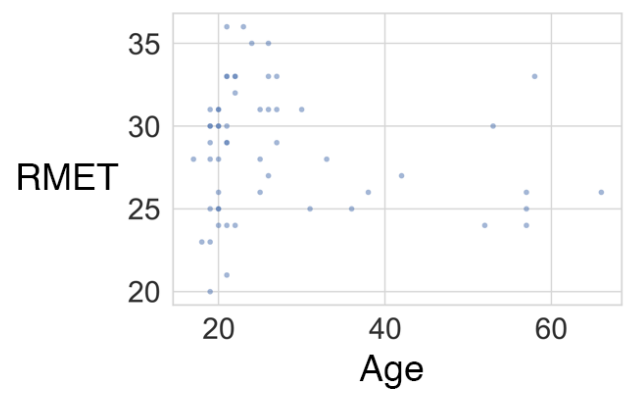

608

609 Figure 10.. Correlation Scatter Plots. A) Autism Quotient and RMET scores B) Autism

610 Quotient scores and Age C) RMET scores and Age

611

612 Furthermore, we performed an analysis to find the electrodes in the EEG set-up that

613 measured the highest ratio of the peak amplitude.

614

615 Table 1. Electrodes with highest ratio of peak amplitude ratio over participants during

616 periodic trials. Noisy electrodes were excluded if they had a R_squared fit of $<0.9$.

\begin{tabular}{|l|l|l|l|l|l|}
\hline $\mathbf{1}$ & FP2 & $\begin{array}{l}\text { ventromedial PreFrontal Cortex } \\
\text { (vmPFC) }\end{array}$ & $\mathbf{6}$ & FP1 & $\begin{array}{l}\text { ventromedial PreFrontal } \\
\text { Cortex (vmPFC) }\end{array}$ \\
\hline 2 & AF8 & $\begin{array}{l}\text { Right Dorsolateral Prefrontal } \\
\text { Cortex }\end{array}$ & $\mathbf{7}$ & FT8 & Orbitofrontal Cortex \\
\hline
\end{tabular}


bioRxiv preprint doi: https://doi.org/10.1101/2020.09.11.292706; this version posted September 12, 2020. The copyright holder for this preprint (which was not certified by peer review) is the author/funder, who has granted bioRxiv a license to display the preprint in perpetuity. It is made available under aCC-BY-NC-ND 4.0 International license.

\section{7}

\begin{tabular}{|l|l|l|l|l|l|}
\hline $\mathbf{3}$ & AF7 & $\begin{array}{l}\text { Left Dorsolateral Prefrontal } \\
\text { Cortex }\end{array}$ & $\mathbf{8}$ & F7 & Left Inferior Frontal \\
\hline $\mathbf{4}$ & T8 & $\begin{array}{l}\text { Right posterior superior } \\
\text { temporal sulcus }\end{array}$ & $\mathbf{9}$ & TP7 & $\begin{array}{l}\text { Left posterior Superior } \\
\text { Temporal Sulcus }\end{array}$ \\
\hline $\mathbf{5}$ & TP8 & $\begin{array}{l}\text { Right posterior Superior } \\
\text { Temporal Sulcus }\end{array}$ & $\mathbf{1 0}$ & P9 & Left Fusiform Face Area \\
\hline
\end{tabular}

618

619 